\title{
Periodic Solutions of Duffing-Type Oscillators Using Continuous Piecewise Linearization Method
}

\author{
Akuro Big-Alabo ${ }^{1}$ \\ ${ }^{1}$ Department of Mechanical Engineering, Faculty of Engineering, University of Port Harcourt, Choba, Rivers State, \\ Nigeria \\ Correspondence: Akuro Big-Alabo, Department of Mechanical Engineering, Faculty of Engineering, University of \\ Port Harcourt, Choba, Rivers State, Nigeria. Tel: 234-816-468-3549. E-mail: akuro.big-alabo@uniport.edu.ng
}

Received: March 24, 2018

Accepted: April 8, 2018

Online Published:May 17, 2018

doi:10.5539/mer.v8n1p41

URL: https://doi.org/10.5539/mer.v8n1p41

\begin{abstract}
A new analytic algorithm called 'continuous piecewise linearization method' (CPLM) is developed to obtain periodic solutions of freely vibrating Duffing-type oscillators. This simple analytic algorithm is based on continuous piecewise linearization of the nonlinear stiffness with respect to displacement and was shown to produce very accurate results for few iterations. The algorithm is valid for Duffing-type oscillators possessing strong nonlinearity and/or undergoing large-amplitude oscillations. Studies conducted on Duffing oscillators with cubic, cubic-quintic and trigonometric sine stiffness nonlinearities showed that the CPLM results match standard numerical solutions and is more accurate than the popular energy balance method (EBM). Additionally, the present analysis shows that the CPLM is capable of predicting the quasi-linear behaviour observed in the oscillation history of Duffing-type oscillators with strongly nonlinearity and/or large-amplitude oscillations. This quasi-linear behaviour cannot be predicted by the EBM to which the CPLM is compared.
\end{abstract}

Keywords: continuous piecewise linearization method, nonlinear systems, Duffing oscillator, large-amplitude oscillations, strong nonlinearity

\section{Introduction}

Conservative systems, which are naturally oscillating undamped systems, are common in nature (e.g. vibration of human eardrum $(\mathrm{He}, 1999 \mathrm{~b})$ ) and in artificially designed systems (e.g. simple pendulum motion). They consist of oscillating systems where the total energy is always constant but is converted from kinetic energy to potential energy and vice versa. The general differential equation governing the oscillations of single degree-of-freedom (SDOF) nonlinear conservative systems can be written as:

$$
\ddot{x}+F(x)=0
$$

with initial conditions: $x(0)=A$ and $\dot{x}(0)=0$; where $F(x)$ is a nonlinear function of $x$ representing the restoring force per unit mass. $F(x)$ may have either linear and nonlinear terms or just nonlinear terms.

A typical nonlinear conservative system that has been the subject of many investigations is the SDOF Duffing-type oscillator. This nonlinear oscillator represents the dynamic behaviour of many practical engineering systems (El-Naggar \& Ismail, 2016) and can be used to investigate important nonlinear phenomena such as hardening or softening response, jumps and bifurcations (Nayfeh \& Mook, 1995). Furthermore, the Duffing-type oscillator is normally used to validate algorithms for obtaining periodic solutions of nonlinear oscillators (He, 1999b; He, 2000; He, 2001; He, 2002; El-Naggar \& Ismail, 2016; Razzak, 2016). The main characteristic of a Duffing-type oscillator is that the nonlinear restoring force is positive when the displacement is positive and vice versa, i.e. $F(+x)>0$ and $F(-x)<0$, as shown in Figure 1(a). This behaviour of the restoring force is different from say a conservative quadratic oscillator where the restoring force is always positive irrespective of whether the displacement is positive or negative i.e. $F( \pm x)>0$. Hence, conservative oscillators such as the quadratic oscillator are not Duffing-type oscillators.

Exact analytical solutions to the vibration of Duffing-type oscillators in the form of equation (1) are generally impossible and therefore, the most accurate periodic solutions are usually obtained numerically. However, approximate analytical techniques to solve equation (1) exist and can be classified as perturbation and non-perturbation methods. Perturbation methods are based on the idea of a completely known linear system in 
equilibrium being subjected to a small disturbance in order to study an unknown nonlinear system. Hence, the classical perturbation methods (see Nayfeh, 1973 for detailed treatment of different classical perturbation methods) depend on a small parameter that has a small range of validity and only produces accurate results for nonlinear systems characterized by small-amplitude oscillations and weak nonlinearity. The recent perturbation methods $(\mathrm{He}$, 1999a; He, 2000; He, 2001; Lui, 2005; El-Naggar \& Ismail, 2016) attempt to deal with the issue of small parameter in order to find solutions that are valid for both small- and large-amplitude oscillations, and weak and strong nonlinearity. In spite of the success of the recent perturbation methods in producing accurate estimates for the nonlinear oscillation frequency, they fail to produce the same success with the oscillation history. Sanchez (2005) demonstrated that the recent perturbation methods perform as poorly as the classical perturbation methods in predicting the oscillation histories of nonlinear systems. Sanchez (2005) further argues that for a solution technique to be considered accurate, it should be capable of accurately predicting both the nonlinear frequency and the displacement and velocity histories. Hence, a perturbation method that can accurately predict the oscillation histories for nonlinear systems with large-amplitude oscillations and/or strong nonlinearity is still lacking and may not be possibly found given the inherent limitation of perturbation methods that they are based on asymptotic series which are divergent for amplitudes greater than one (He, 2005).

On the other hand, the non-perturbation methods do not use any small or artificial parameter. Examples include variational iteration method - VIM (He, 1999b), EBM (He, 2002), He-Chengtian interpolation method (He, 2006) and Adomain decomposition method - ADM (Adomian, 1988). The non-perturbation methods also have their own limitations when used to find periodic solutions for nonlinear oscillators. For instance, Zhang et al (2017) showed that the ADM may sometimes have convergence issues in terms of rate of convergence and converging to an accurate solution. Hence, the authors suggested an improved decomposition method that attempts to address the observed limitations of ADM by means of an optimal convergence acceleration parameter. Also, it has been observed that higher-order approximations of existing non-perturbation methods do not always improve the accuracy of the predicted oscillation history (He, 2006). Another challenge in using the existing non-perturbation methods is that the solution procedure becomes increasingly complex for higher-order approximations and in some cases the algebraic manipulation required is practically prohibitive (Lim et al., 2005; Shariyat \& Darabi, 2013) unless conducted with the aid of sophisticated programs capable of handling difficult algebraic computations e.g. Mathematica ${ }^{\mathrm{TM}}$ and Maple. Consequently, some of the non-perturbation methods are normally applied for the initial approximation (He, 2002; $\mathrm{He}, 2006)$ and this limits the accuracy of the solution obtained. Also, some non-perturbation methods are heuristic $(\mathrm{He}, 1999 \mathrm{~b} ; \mathrm{He}, 2002)$ and require experience to choose an appropriate initial trial function.

The present study proposes a new iterative analytic algorithm to address the issues of solution accuracy, solution complexity and limited range of validity observed in existing perturbation and non-perturbation methods. This study presents a simple analytic algorithm for the periodic solution of Duffing-type oscillators in the form of equation (1). The algorithm is based on the idea of continuous piecewise linearization of the nonlinear stiffness and is capable of accurately predicting the oscillation histories and nonlinear frequency of Duffing-type oscillators characterized by strong nonlinearity and/or large-amplitude oscillations.
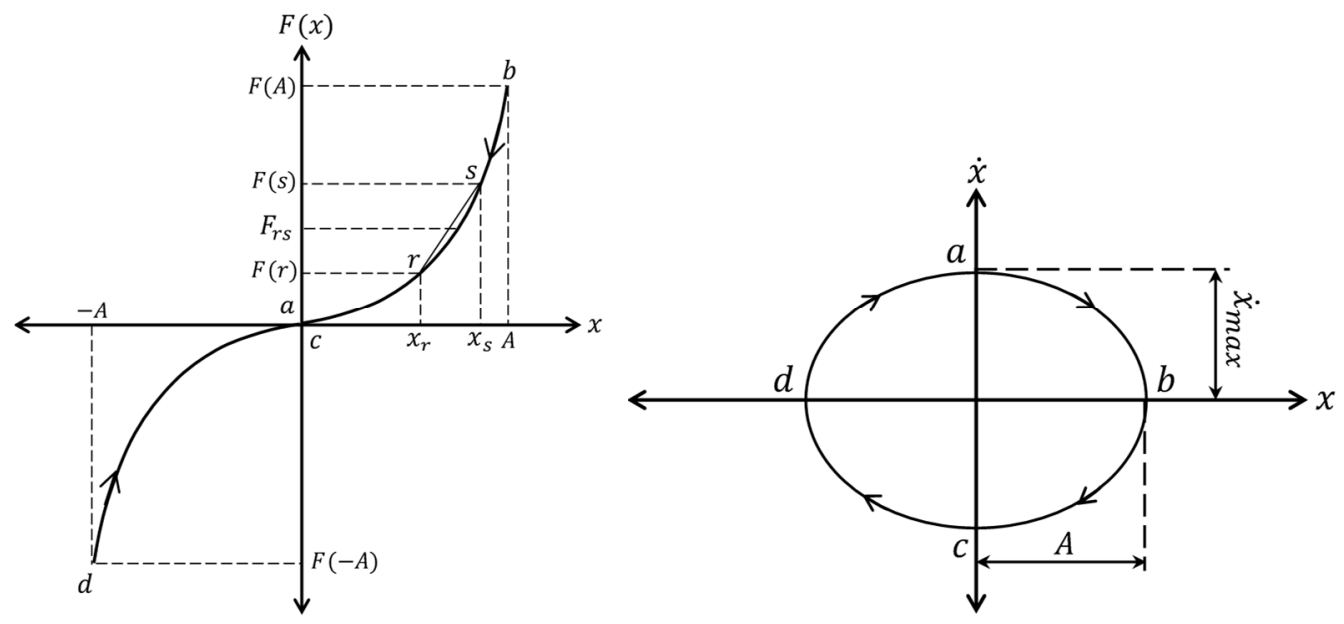

Figure 1. (a) Typical compliance curve for Duffing-type spring and (b) phase plot of oscillations 


\section{Concept and Mathematical Formulation of the Present Algorithm}

The fundamental principle of the present algorithm is the continuous piecewise linearization of the nonlinear stiffness (or restoring force), resulting to linear ODEs each of which produces a solution that covers a time-range of the oscillation history. Therefore, the present algorithm is called continuous piecewise linearization method (CPLM). This principle was first applied to develop an algorithm called 'force-indentation linearisation method' (FILM) that was specifically designed to solve the nonlinear models of a half-space impact system (Big-Alabo et al., 2015). However, the half-space impact system is not an oscillating system because the displacement and excitation (i.e. impact force) are non-oscillatory and are restricted as follows: $x \geq 0$ and $F_{\text {impact }} \geq 0$ where $x$ is a positive displacement called indentation. For nonlinear oscillators, this restriction has to be removed because the displacement and/or excitation can be positive or negative. This means that the FILM cannot be used to produce periodic solutions for nonlinear oscillating systems. Therefore, the piecewise linearization technique of the FILM is adapted here to formulate the CPLM which can provide periodic solutions for nonlinear Duffing-type oscillators.

The compliance characteristics of the nonlinear restoring force for a Duffing-type oscillator and the corresponding phase-plot are illustrated in Figure 1. It is noteworthy that for the Duffing-type oscillator shown in Figure 1a the restoring force increases monotonically to a maximum when the displacement is positive (i.e. $0 \leq F \leq F(A)$ for $0 \leq x \leq A$ ) and vice versa (i.e. $F(-A) \leq F \leq 0$ for $-A \leq x \leq 0$ ). In Figure 1a, $F_{r s}$ is the restoring force for the discretization bounded by points $r$ and $s$, and can be approximated using a linearized restoring force if $\Delta x=x_{s}-$ $x_{r}$ is sufficiently small. Details of the discretization and linearization technique can be found in Big-Alabo et al. $(2015,2017)$ and are not repeated here for brevity. The main goal is to apply the discretization and linearization concepts to develop the CPLM, which can provide periodic solutions for Duffing-type oscillators. Following the linearization technique in Big-Alabo et al. (2017), the linearized restoring force for each $n$ discretization of the nonlinear restoring force in equation (1) can be expressed as:

$$
F_{r s}(x)=K_{r s}\left(x-x_{r}\right)+F_{r}
$$

where $K_{r s}=\left|\left[F\left(x_{s}\right)-F\left(x_{r}\right)\right] /\left(x_{s}-x_{r}\right)\right|$ is the linearized stiffness, and $F_{r}=F\left(x_{r}\right)$. Also, $r=0,1,2,3, \ldots, n-1$ and $s=r+1$.

Substituting equation (2) in (1) gives the linear ODE for each discretization as:

$$
\ddot{x}+K_{r s} x=K_{r s} x_{r}-F_{r}
$$

The displacement and velocity are respectively obtained from equation (3) as shown below.

$$
\begin{gathered}
x(t)=R_{r s} \sin \left(\omega_{r s} t+\varphi_{r s}\right)+C_{r s} \\
\dot{x}(t)=\omega_{r s} R_{r s} \cos \left(\omega_{r s} t+\varphi_{r s}\right)
\end{gathered}
$$

The constants in equations (4) and (5) are calculated as: $R_{r s}=\left[\left(x_{r}-C_{r s}\right)^{2}+\left(\dot{x}_{r} / \omega_{r s}\right)^{2}\right]^{1 / 2}, \omega_{r s}=\sqrt{K_{r s}}$, and $C_{r s}=x_{r}-F\left(x_{r}\right) / K_{r s}$. The initial conditions and other parameters are determined based on the stage of the oscillation. For oscillations from point $b$ to point $d$ through point $c$ (see Figure 1b) the initial conditions for each discretization are $x_{r}(0)=A-r \Delta x$ and $\dot{x}_{r}(0)=-\sqrt{\left|2 \int_{A}^{x_{r}}-F(x) d x\right|}$; where $\Delta x=A / n$ and the other parameters are calculated as:

$$
\begin{gathered}
\varphi_{r s}=\left\{\begin{array}{ll}
0.5 \pi & \dot{x}_{r}=0 \\
\pi+\tan ^{-1}\left[\omega_{r s}\left(x_{r}-C_{r s}\right) / \dot{x}_{r}\right] & \dot{x}_{r}<0
\end{array} ;\right. \text { and } \\
\Delta t=\left\{\begin{array}{ll}
\left(0.5 \pi-\varphi_{r s}\right) / \omega_{r s} & \left(x_{s}-C_{r s}\right) \geq R_{r s} \\
\left(0.5 \pi+\cos ^{-1}\left[\left(x_{s}-C_{r s}\right) / R_{r s}\right]-\varphi_{r s}\right) / \omega_{r s} & \left(x_{s}-C_{r s}\right)<R_{r s}
\end{array} .\right.
\end{gathered}
$$

For oscillations from point $d$ to point $b$ through point $a$ (see Figure 1b) the initial conditions for each discretization are $x_{r}(0)=-A+r \Delta x$ and $\dot{x}_{r}(0)=\sqrt{\left|2 \int_{A}^{x_{r}}-F(x) d x\right|}$, whereas the other parameters are calculated as:

$$
\varphi_{r s}=\left\{\begin{array}{ll}
-0.5 \pi & \dot{x}_{r}=0 \\
\tan ^{-1}\left[\omega_{r s}\left(x_{r}-C_{r s}\right) / \dot{x}_{r}\right] & \dot{x}_{r}>0
\end{array} ;\right. \text { and }
$$




$$
\Delta t=\left\{\begin{array}{ll}
\left(0.5 \pi-\varphi_{r s}\right) / \omega_{r s} & \left(x_{s}-C_{r s}\right) \geq R_{r s} \\
\left(0.5 \pi-\cos ^{-1}\left[\left(x_{s}-C_{r s}\right) / R_{r s}\right]-\varphi_{r s}\right) / \omega_{r s} & \left(x_{s}-C_{r s}\right)<R_{r s}
\end{array} .\right.
$$

Finally, the time at the end of each discretization is $t_{s}=t_{r}+\Delta t$ and the end conditions $x_{s}$ and $\dot{x}_{s}$ are calculated by replacing $r$ with $s$ in the formulae for initial conditions. The above method is used to develop the flowcharts in Figures 2 and 3 for easy implementation in computer programming. The flowcharts demonstrate the algorithm for oscillations from point $b$ to point $d$ through point $c$ but similar flowcharts are applicable for oscillations from point $d$ to point $b$ through point $a$.

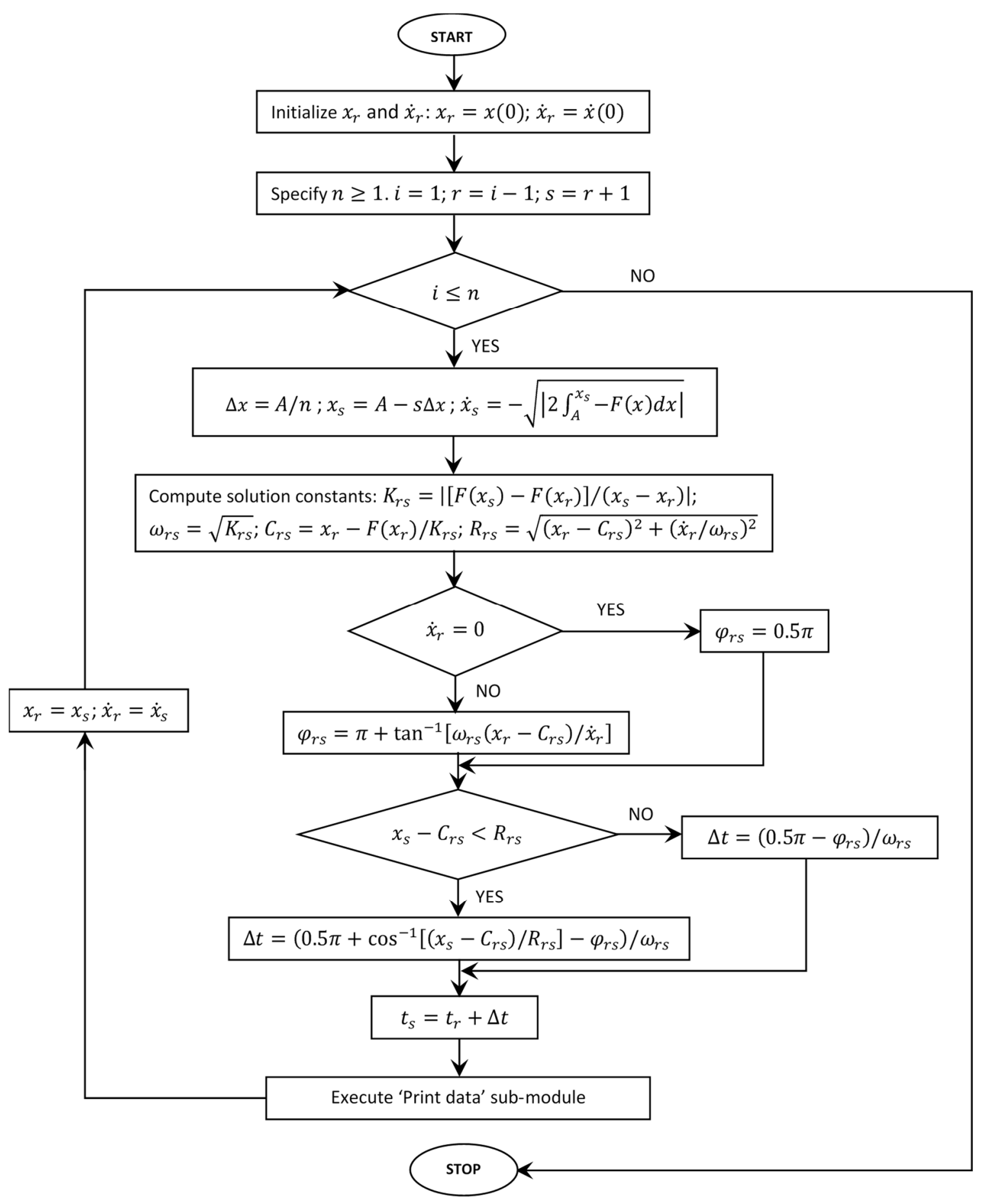

Figure 2. Flowchart for implementing the CPLM for oscillations from point $b$ to point $d$ through point $c$ 
A basic difference between the existing approximate analytical schemes for solution of nonlinear systems and the CPLM is in the solution concept. The other analytical schemes start with an initial solution, which is usually based on a linear approximation of the nonlinear ODE, and then try to improve on the initial solution using higher-order approximations. The CPLM assumes no initial solution but uses an approximation technique, i.e. piecewise discretization and linearization of the nonlinear stiffness, which deals directly with the nonlinear ODE at every stage of its oscillation history. In addition, the CPLM does not use any small or artificial parameter and higher-order approximations are not required to improve the accuracy of the solution. Hence, the limitations associated with the use of special parameters or higher-order approximations are completely avoided by the CPLM and yet it remains simple in its formulation and implementation.

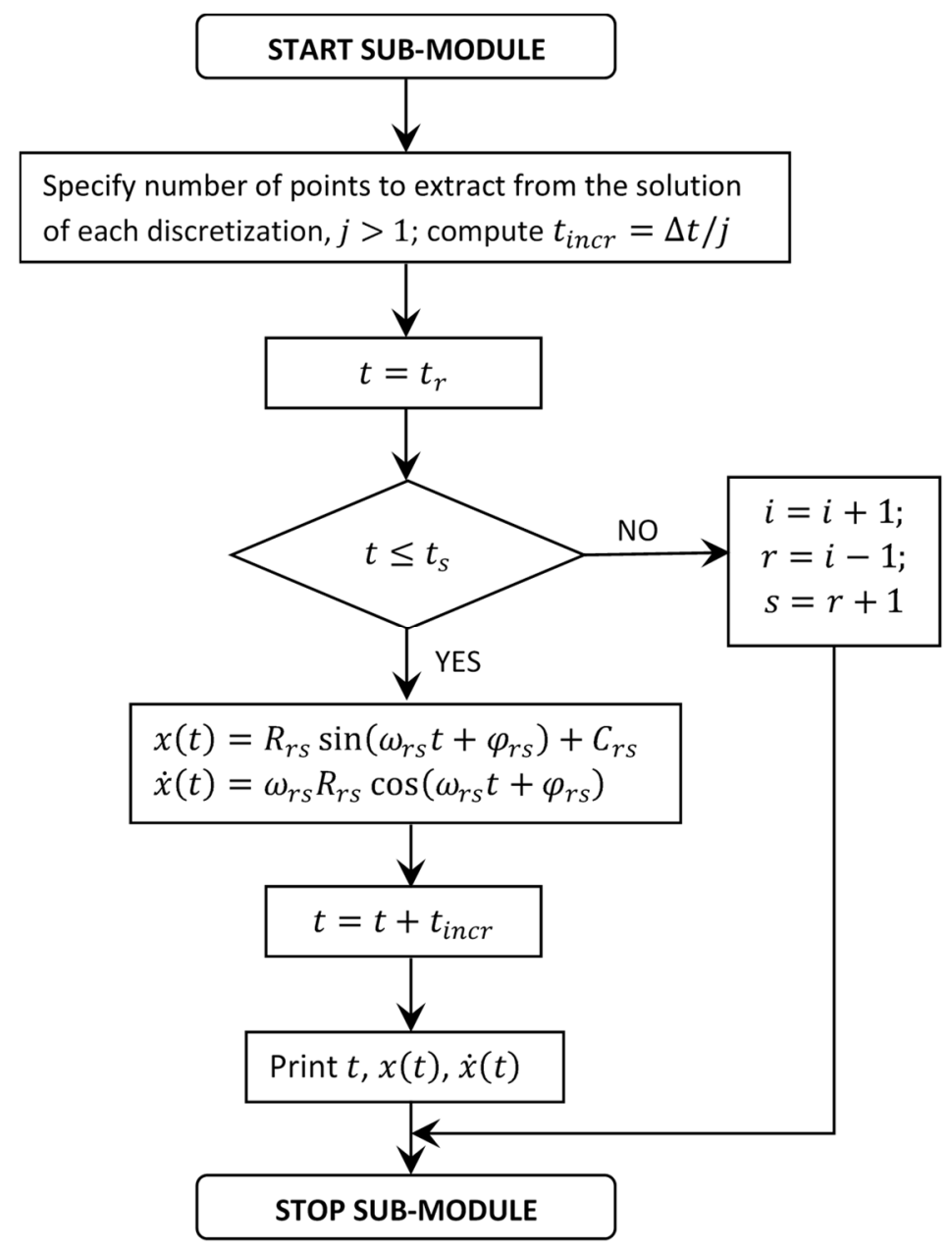

Figure 3. Flowchart for sub-module to print data from the solution of each discretization

It is necessary to mention that the CPLM and the FILM are very similar in their formulation because both are based on the same piecewise linearization concept. Therefore the CPLM shares in all the advantages of the FILM. However, the CPLM is different from the FILM in the sense that the CPLM is formulated to solve nonlinear oscillating systems while the FILM cannot. In other words, the CPLM may be viewed as a modification of the FILM to enable periodic solutions of nonlinear oscillating systems but has not been called modified-FILM because the displacement considered is not an indentation.

\section{Numerical Validation of CPLM for Duffing-Type Oscillators}

In this section the CPLM algorithm is validated by considering three common examples of conservative Duffing-type oscillators with the following nonlinearities: cubic, cubic-quintic and trigonometric sine. 


\subsection{Duffing-Type Oscillator with Cubic Nonlinearity}

To investigate the validity of the CPLM we consider the Duffing oscillator with cubic nonlinear stiffness as shown:

$$
\ddot{x}+k_{1} x+k_{2} x^{3}=0
$$

The initial conditions are: $x(0)=A$ and $\dot{x}(0)=0$, and the restoring force is $F(x)=k_{1} x+k_{2} x^{3}$. Equation (6) was solved for $k_{1}=k_{2}=1$ and $A=0.5$ using the CPLM and numerical method. The numerical solution was obtained using the 'StiffnessSwitching' algorithm of the NDSolve function in Mathematica ${ }^{\mathrm{TM}}$, and the results of the numerical solution are considered to be the standard results i.e. exact solutions. The error estimates in the time period predicted by the CPLM for different values of $n$ are shown in Table 1. It is observed that the error decreases very rapidly as $n$ increases and very good accuracy is obtained for a few discretization. Also, Table 1 shows the time period predicted by the EBM and it is observed that for this example the EBM produced the same order of error as the CPLM when $n=5$.

Table 1. Error analysis in the estimation of time period by the CPLM

\begin{tabular}{lcccccccc}
\hline & & \multicolumn{1}{c}{ CPLM } \\
& Numerical & EBM & $\boldsymbol{n}=\mathbf{1}$ & $\boldsymbol{n}=\mathbf{2}$ & $\boldsymbol{n}=\mathbf{3}$ & $\boldsymbol{n}=\mathbf{4}$ & $\boldsymbol{n}=\mathbf{5}$ & $\boldsymbol{n}=\mathbf{2 5}$ \\
\hline Period & 5.76919 & 5.76584 & 5.61984 & 5.73764 & 5.75660 & 5.76260 & 5.76516 & 5.76876 \\
Error (\%) & - & 0.058 & 2.589 & 0.547 & 0.218 & 0.114 & 0.070 & 0.007 \\
\hline
\end{tabular}

In line with the validation benchmark of Sanchez (2005) additional validation of the CPLM was conducted by comparing the oscillations as shown in Figure 4. The figure shows that the CPLM solution for $n=5$ and that of the EBM are in good agreement with the numerical solution in the case of small-amplitude oscillations of a weakly nonlinear cubic Duffing oscillator. Also, the figure confirms the remarkable accuracy of the CPLM for few iterations (or discretization) i.e. $n=5$.

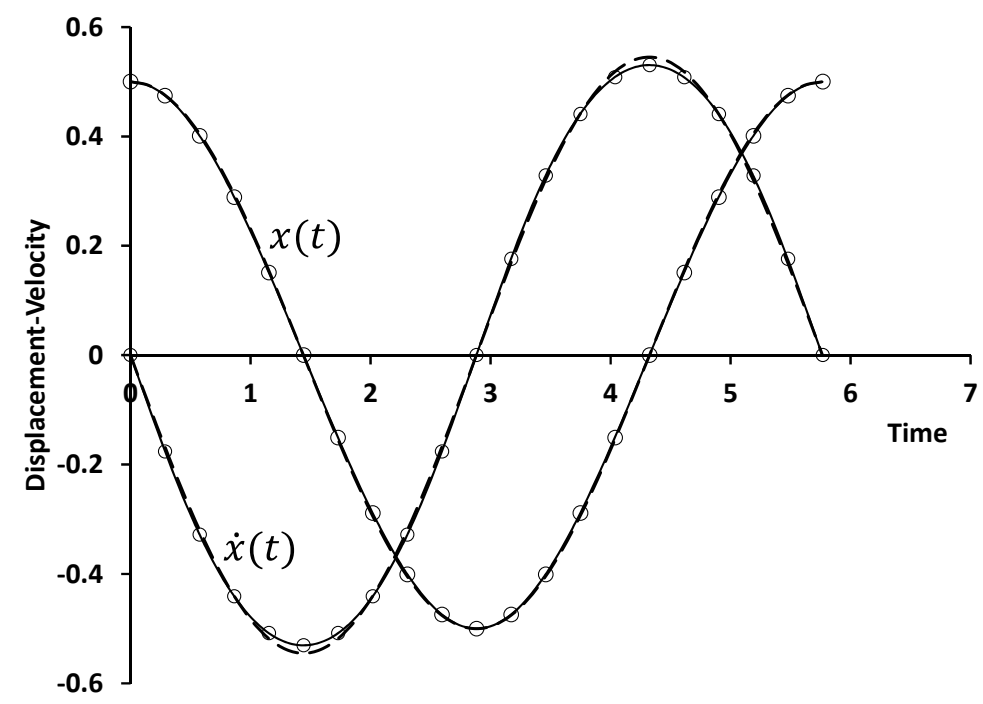

Figure 4: Displacement and velocity history for cubic Duffing oscillator (equation (6)) with weak nonlinearity and small-amplitude oscillations; $k_{1}=k_{2}=1$ and $A=0.5$. Continuous line-CPLM, Dash line-EBM, CircleNumerical solution

\subsection{Duffing-Type Oscillator with Cubic-Quintic Nonlinearity}

The dynamic equation for the Duffing-type oscillator with cubic and quintic nonlinear stiffness is given by (Razzak, 2016):

$$
\ddot{x}+k_{1} x+k_{2} x^{3}+k_{3} x^{5}=0
$$

The initial conditions are: $x(0)=A$ and $\dot{x}(0)=0$, and the restoring force is $F(x)=k_{1} x+k_{2} x^{3}+k_{3} x^{5}$. Razzak (2016) obtained approximate analytical periodic solutions for equation (7) using a first-order variational approach. Razzak's solution for the oscillation history is the same as the EBM solution and is expressed as: 


$$
x=A \cos \omega t
$$

where $\omega$ is the nonlinear oscillation frequency. Razzak (2016) obtained $\omega$ for equation (7) through variational approach as:

$$
\omega=\sqrt{k_{1}+\frac{79 k_{2} A^{2}}{106}+\frac{437 k_{3} A^{4}}{848}}
$$

The oscillations of the cubic-quintic Duffing oscillator was simulated for $k_{1}=k_{2}=k_{3}=1$ and $A=1.0$ as shown in Figure 5. This case study represents a weakly nonlinear cubic-quintic Duffing oscillator undergoing moderate oscillations and has been investigated previously (Razzak, 2016). Figure 5 shows that the oscillations predicted by the CPLM for $n=5$ are in good agreement with the numerical solution. The EBM and Razzak's variational solution are close to the numerical solution for the displacement history but show significant deviations around the turning points of the velocity history. Also, the CPLM time period estimate when $n=25$ (4.124) is more accurate than those of Razzak's variational approach (4.179) and EBM (4.113) when compared with the exact time period (4.124).

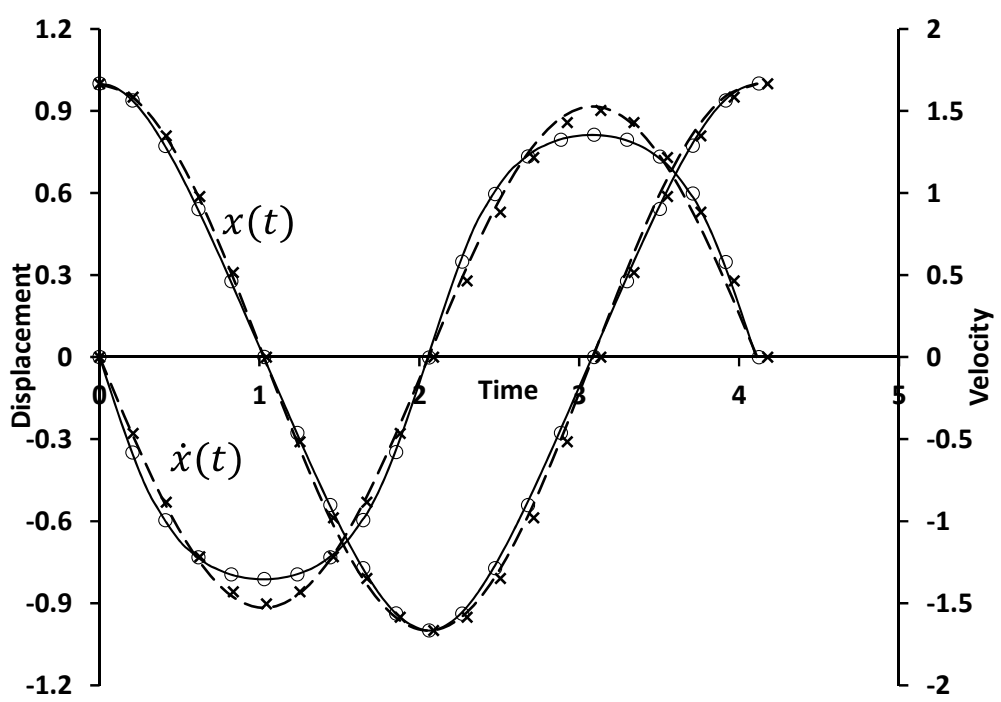

Figure 5. Displacement and velocity history for cubic-quintic Duffing oscillator (equation (7)) with weak nonlinearity and moderate-amplitude oscillations; $k_{1}=k_{2}=k_{3}=1$ and $A=1.0$. Continuous line - CPLM, Dash line - EBM, Cross - Variational solution, Circle - Numerical solution

Note that the EBM frequency solution for equation (7) can be derived for a collocation point of $\omega t=\pi / 4$ as:

$$
\omega=\sqrt{k_{1}+\frac{3 k_{2} A^{2}}{4}+\frac{7 k_{3} A^{4}}{12}}
$$

\subsection{Moderate-Amplitude Oscillations of a Simple Pendulum}

An important physical system for studying nonlinear phenomena is the simple pendulum. The dynamic model for the oscillations of the simple pendulum after neglecting air resistance can be written as:

$$
\ddot{x}+\Omega^{2} \sin x=0
$$




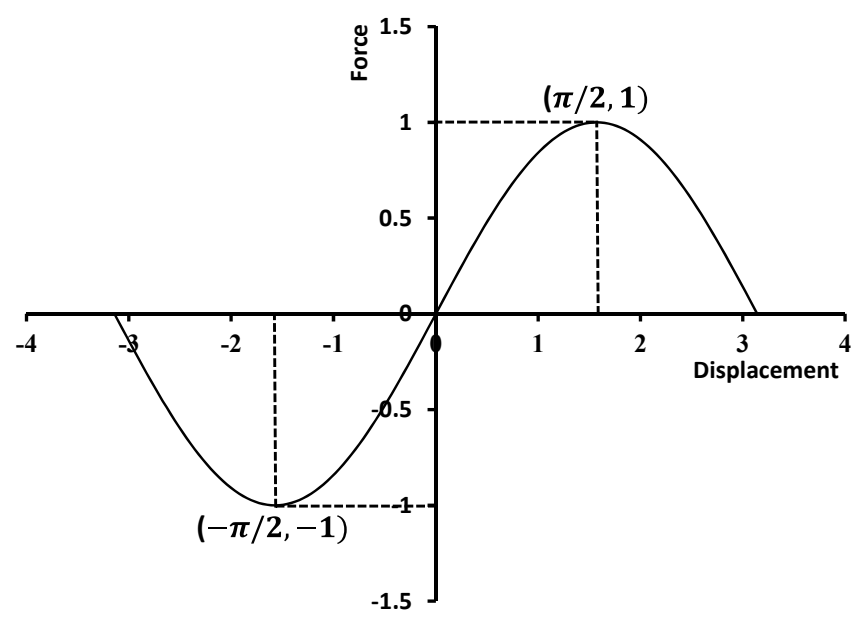

Figure 6. Compliance curve for motion of simple pendulum within the range of $-\pi \leq x \leq \pi$

with initial conditions $x(0)=A$ and $\dot{x}(0)=0$, where $x$ is the angular displacement and $\Omega=\sqrt{g / l}$ is the nonlinear stiffness constant which is equal to the natural frequency for small oscillations of a simple pendulum. The compliance curve for this oscillator (see Figure 6) shows that the simple pendulum only exhibits Duffing-type oscillations for displacements in the range $-\pi / 2 \leq x \leq \pi / 2$. Therefore, the CPLM can be applied to obtain periodic solutions for the nonlinear oscillations of a simple pendulum undergoing small- $\left(|x| \leq 0.175 \approx 10^{\circ}\right)$ to moderate-amplitude $(0.175<|x| \leq 1.571)$ oscillations. Figure 7 shows the oscillations of the simple pendulum when $\Omega=1.0$ and $A=1.5$. The EBM frequency-amplitude solution for equation (11) has been derived by $\mathrm{He}$ (2002) as $\omega=\Omega \sqrt{2(1-\cos A) / A^{2}}$, and this expression was used in simulating the EBM solution for this example. An excellent agreement is observed between the pendulum oscillations predicted by the CPLM for $n=20$ and the numerical solution, while the EBM shows significant errors in its prediction of the pendulum oscillations. Also, the CPLM time period estimate for $n=25$ (7.289) is more accurate than that of the EBM (6.913) when compared to the exact time period (7.299).

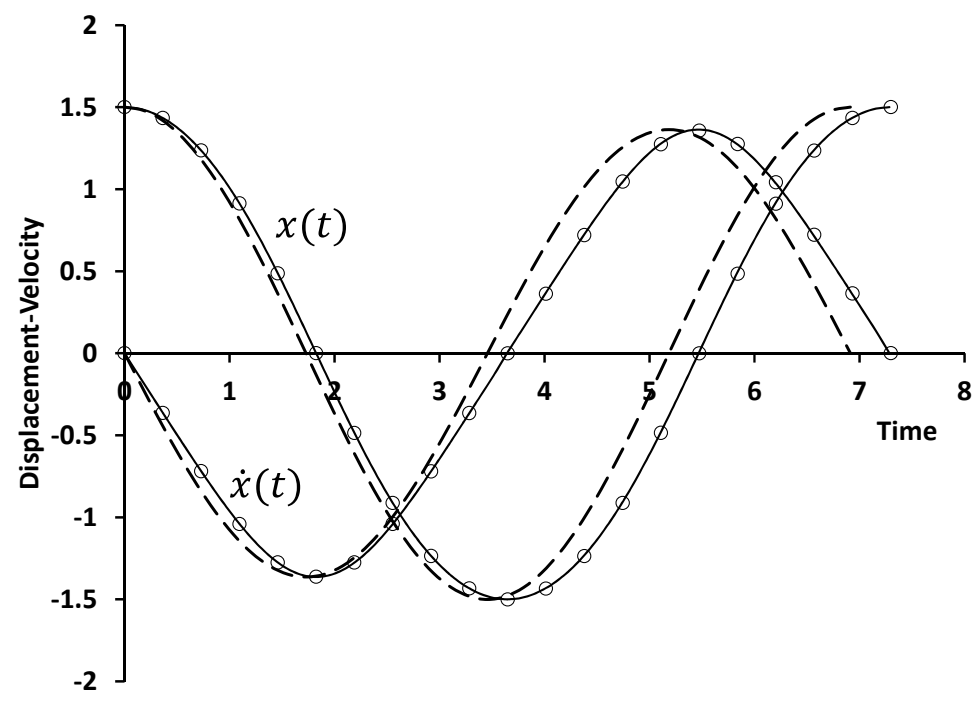

Figure 7: Displacement and velocity history for pendulum motion (equation (11)) with weak nonlinearity and moderate-amplitude oscillations; $\Omega=1$ and $A=1.5$. Continuous line - CPLM, Dash line - EBM, Circle Numerical solution 


\section{Duffing Oscillator with Large-Amplitude Oscillations}

In order to test the effectiveness of the CPLM to predict large-amplitude oscillations of nonlinear systems the cubic Duffing oscillator of equation (6) was re-examined for large values of $A$. The oscillations predicted by the CPLM when $n=5$ were compared with results of numerical solutions and EBM predictions for $k_{1}=k_{2}=1$ and for $A=10,100$ as shown in Figures 8a and 8b. Also, estimates of the time period for the considered amplitudes are shown in Table 2. These results demonstrate that the CPLM performs excellently well in predicting the large-amplitude oscillations of the cubic Duffing oscillator.

Table 2. Time period for cubic Duffing oscillator subjected to large-amplitude oscillations

\begin{tabular}{lcccccc}
\hline & \multicolumn{3}{c}{$\boldsymbol{k}_{\mathbf{1}}=\boldsymbol{k}_{\mathbf{2}}=\mathbf{1} ; \boldsymbol{A}=\mathbf{1 0}$} & \multicolumn{3}{c}{$\boldsymbol{k}_{\mathbf{1}}=\boldsymbol{k}_{\mathbf{2}}=\mathbf{1} ; \boldsymbol{A}=\mathbf{1 0 0}$} \\
\hline Numerical & $\mathbf{E B M}$ & $\mathbf{C P L M} \boldsymbol{n}=\mathbf{2 5}$ & Numerical & EBM & CPLM $\boldsymbol{n}=\mathbf{2 5}$ \\
\hline Erriod & 0.73629 & 0.72073 & 0.73624 & 0.074157 & 0.072547 & 0.074153 \\
& - & 2.113 & 0.007 & - & 2.172 & 0.005 \\
\hline
\end{tabular}

It was observed from the results of the CPLM and numerical solution (Figures $8 \mathrm{a}$ and $8 \mathrm{~b}$ ) that the velocity is almost constant in the region of its turning point. The implication is that the displacement history exhibits a quasi-linear behaviour in the same region. This qualitative behaviour of the cubic Duffing oscillator experiencing large-amplitude oscillations was adequately captured by the CPLM and the numerical solution, but was not captured by the EBM. This is because the EBM solution for the oscillations is based on trigonometric functions that have no quasi-linear characteristics (see equation (8)). Therefore, the EBM produced significant errors in predicting the large-amplitude oscillations of equation (6); especially the velocity history. Note that this inherent limitation in the oscillation history of the EBM is also found in the variational solution proposed by Razzak (2016).

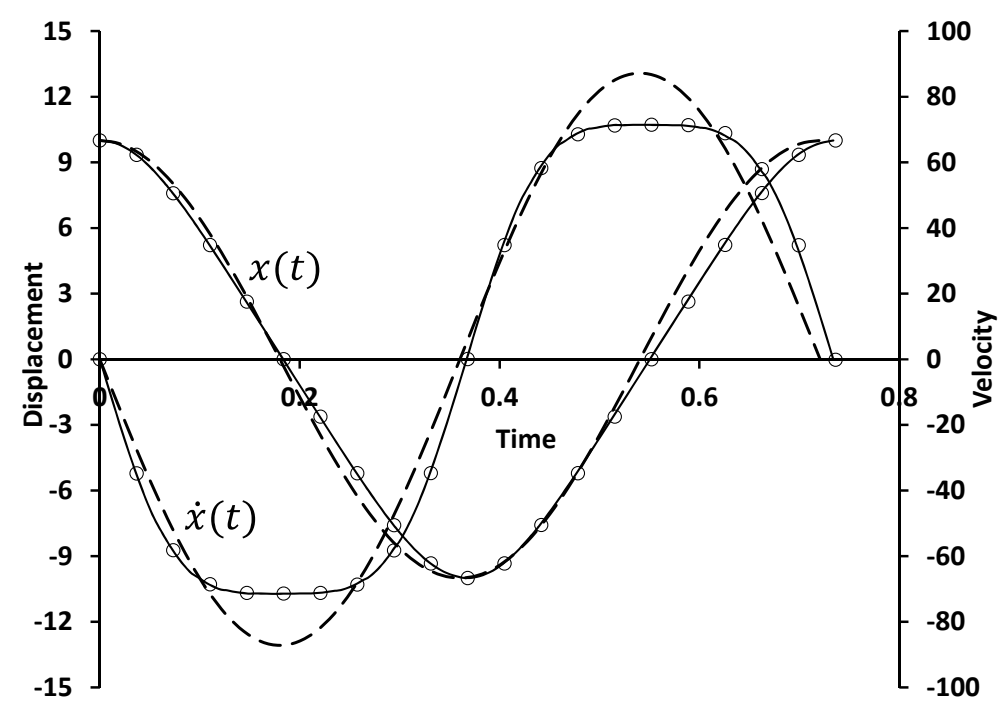

Figure 8a. Displacement and velocity history for cubic Duffing oscillator (equation (6)) with large-amplitude oscillations; $k_{1}=k_{2}=1$ and $A=10$. Continuous line-CPLM, Dash line-EBM, Circle-Numerical solution 


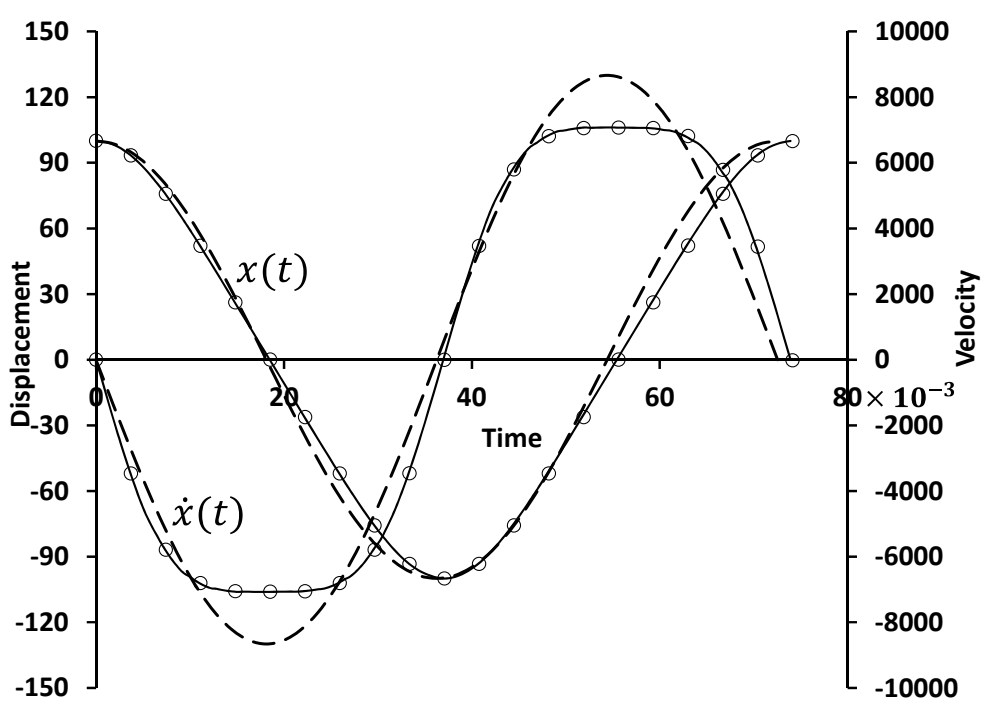

Figure 8b. Displacement and velocity history for cubic Duffing oscillator (equation (6)) with large-amplitude oscillations; $k_{1}=k_{2}=1$ and $A=100$. Continuous line-CPLM, Dash line - EBM, Circle - Numerical solution

\section{Duffing Oscillator Possessing Strong Nonlinearity}

For the case of a Duffing-type oscillator with strong nonlinearity (i.e. stiff nonlinear systems) the cubic Duffing oscillator of equation (6) was re-examined for a large value of the nonlinear stiffness constant, $k_{2}$. Moderate- and large-amplitude oscillations of a strongly nonlinear cubic Duffing oscillator were investigated. The predicted oscillations are shown in Figures 9a and 9b while the time period estimates are given in Table 3. Again, the strongly nonlinear cubic Duffing oscillator exhibits a quasi-linear behaviour that was accurately predicted by the CPLM but not the EBM.

Table 3. Time period for cubic Duffing oscillator with strong nonlinearity

\begin{tabular}{lcccccc}
\hline & \multicolumn{3}{c}{$\boldsymbol{k}_{\mathbf{1}}=\mathbf{1} ; \boldsymbol{k}_{\mathbf{2}}=\mathbf{1 0 0 0} ; \boldsymbol{A}=\mathbf{1}$} & \multicolumn{3}{c}{$\boldsymbol{k}_{\mathbf{1}}=\mathbf{1} ; \boldsymbol{k}_{\mathbf{2}}=\mathbf{1 0 0 0} ; \boldsymbol{A}=\mathbf{1 0}$} \\
\hline Numerical & $\mathbf{E B M}$ & $\mathbf{C P L M} \boldsymbol{n}=\mathbf{2 5}$ & Numerical & EBM & CPLM $\boldsymbol{n}=\mathbf{2 5}$ \\
\hline Erriod & 0.23435 & 0.22928 & 0.23434 & 0.023452 & 0.022943 & 0.023451 \\
& - & 2.166 & 0.004 & - & 2.172 & 0.004 \\
\hline
\end{tabular}

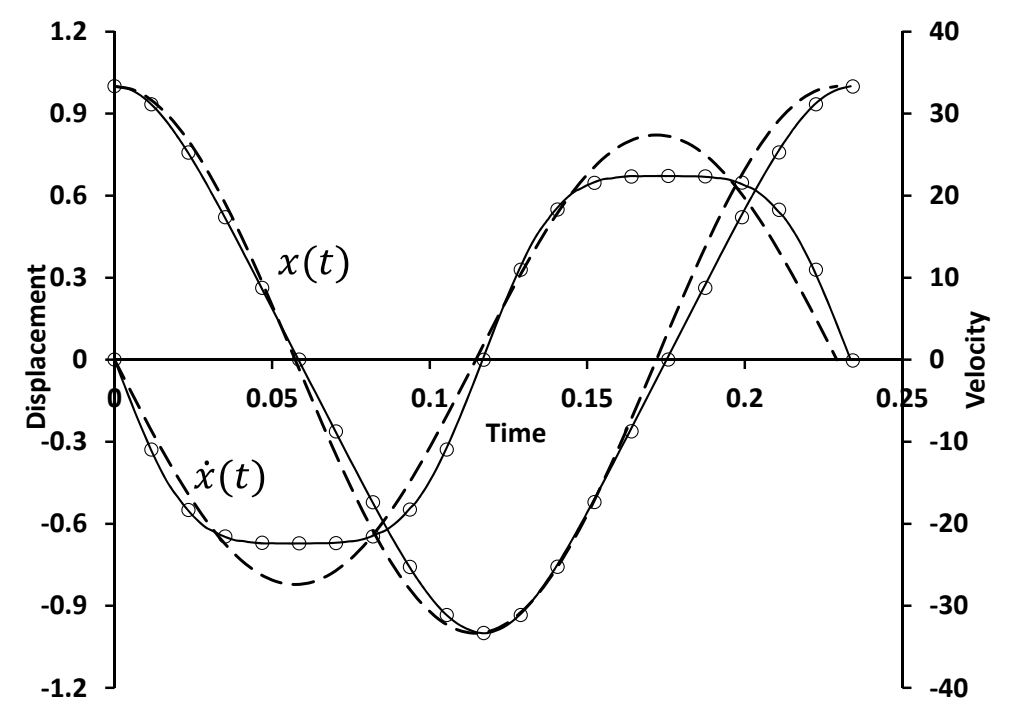

Figure 9a. Displacement and velocity history for Duffing oscillator (equation (6)) with strong nonlinearity and small-amplitude oscillations; $k_{1}=1 ; k_{2}=1000$ and $A=1$. Continuous line-CPLM, Dash line - EBM, Circle - Numerical solution 


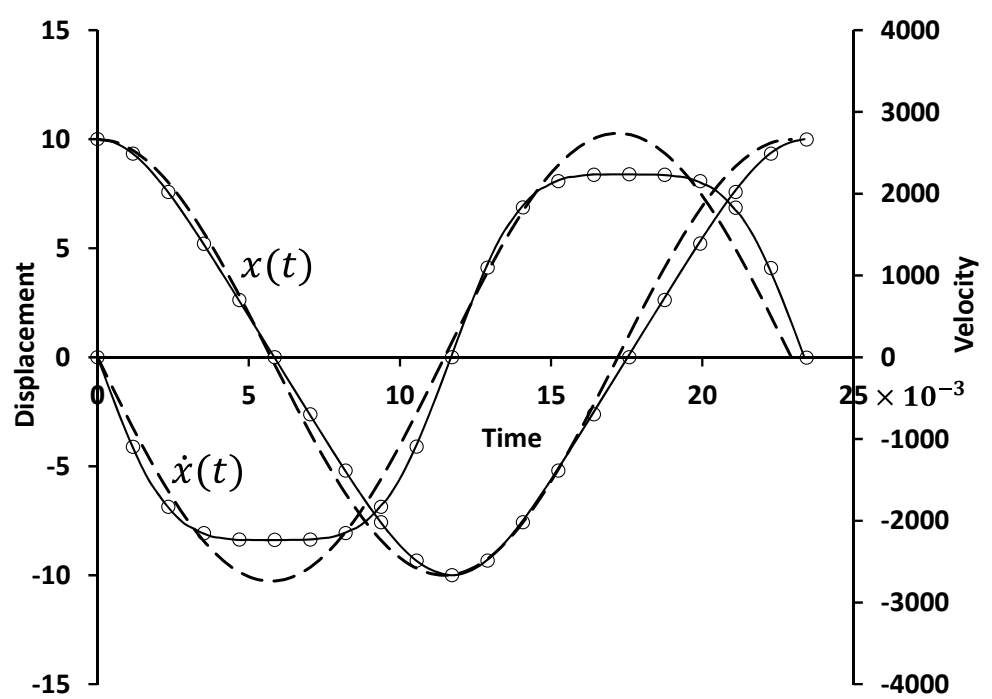

Figure 9b. Displacement and velocity history for cubic Duffing oscillator (equation (6)) with strong nonlinearity and large-amplitude oscillations; $k_{1}=1 ; k_{2}=1000$ and $A=10$. Continuous line-CPLM, Dash line - EBM, Circle

- Numerical solution

The results in Figures 8 and 9 are the same qualitatively but differ quantitatively. When the amplitude of the displacement is increased by an order of 10 the amplitude of the velocity is increased by two orders of 10 while the time period is decreased by an order of 10. Furthermore, Figures 8 and 9 suggest that the quasi-linear effect of a nonlinear Duffing-type oscillator becomes evident during large-amplitude oscillations or for large values of the nonlinear stiffness constant. The ability of a solution algorithm to predict such qualitative behaviour in nonlinear systems in crucial for adequate understanding of nonlinear effects. This underscores the need for a solution algorithm to accurately predict the oscillation histories and not just the nonlinear frequency. Therefore, the CPLM is presented as an adequate solution method for the analysis of conservative Duffing-type oscillators.

\section{Conclusions}

A novel and simple analytic algorithm that is based on continuous piecewise linearization of the nonlinear stiffness has been presented for the solution of Duffing-type oscillators with nonlinear stiffness (or restoring force). The algorithm, which is called continuous piecewise linearization method, converges very fast to the exact solution. The method was shown to be reasonably accurate for as few as five iterations (or discretization) and is capable of accurately predicting the oscillations of Duffing-type systems with strong nonlinearity or undergoing large-amplitude oscillations. In addition to its simplicity, another attractive feature of the CPLM is that its implementation remains easy irrespective of the complexity of the nonlinear restoring force. Just like the FILM, the CPLM is also inherently stable and does not produce divergent solutions. The CPLM shows potential and may be extended to handle other types of nonlinear conservative oscillators that are not Duffing-type oscillators.

\section{Acknowledgments}

Dr C.V. Ossia of the Department of Mechatronic Engineering, University of Port Harcourt, Nigeria is gratefully acknowledged for his suggestions on improving the paper. This research is not funded.

\section{References}

Adomian, G. A. (1988). Review of the decomposition method in applied mathematics, Journal of Mathematical Analysis and Applications, 135, 501-544. https://doi.org/10.1016/0022-247X(88)90170-9

Big-Alabo, A., Cartmell, M. P., \& Harrison, P. (2017). On the solution of asymptotic impact problems with significant localised indentation, Journal of Mechanical Engineering Sciences, 231(5), 807-822. https://doi.org/10.1177/ 0954406216628556

Big-Alabo, A., Harrison P., \& Cartmell, M. P. (2015). Algorithm for the solution of elastoplastic half-space impact. Force-Indentation Linearisation Method, Journal of Mechanical Engineering Sciences, 229(5), 850-858. https://doi.org/10.1177/0954406214541431

El-Naggar, A. M., \& Ismail, G. M. (2016). Analytical solution of strongly nonlinear Duffing oscillators, Alexandria Engineering Journal, 55, 2959-2965. http://dx.doi.org/10.1016/j.aej.2015.07.017 
He, J. H. (1999a). Homotopy perturbation technique. Computer Methods in Applied Mechanics and Engineering, 178(3/4), 257-262. https://doi.org/10.1016/S0045-7825(99)00018-3.

He, J. H. (1999b). Variational iteration method: a kind of nonlinear analytical technique: some examples. International Journal of Nonlinear Mechanics, 34(4), 699-708. https://doi.org/10.1016/S0020-7462(98)00048-1

He, J. H. (2000). A new perturbation technique which is also valid for large parameters. Journal of Sound and Vibration, 229(5), 1257-1263. https://doi.org/10.1006/jsvi.1999.2509

He, J. H. (2001). Iteration perturbation method for strongly nonlinear oscillations. Journal of Vibration and Control, 7(5), 631-642. https://doi.org/10.1177/107754630100700501.

He, J. H. (2002). Preliminary report on the energy balance for nonlinear oscillations. Mechanics Research Communications, 29, 107-111. https://doi.org/10.1016/S0093-6413(02)00237-9.

He, J. H. (2005). Author's reply to 'A view to the new perturbation technique valid for large parameters' by N.E. Sanchez. Journal of Sound and Vibration, 282, 1317-1320. https://doi.org/10.1016/j.jsv.2004.09.030

He, J. H. (2006). Some asymptotic methods for strongly nonlinear equations. International Journal of Modern Physics B, 20(10), 1141-1199. https://doi.org/10.1142/S0217979206033796

Lim, C. W., Lai, S. K., \& Wu, B. S. (2005). Accurate higher-order analytical approximate solution to large-amplitude oscillating systems with a general non-rational restoring force. Nonlinear Dynamics, 42, 267-281. https://doi.org/10.1007/s11071-005-4025-z

Lui, H. M. (2005). Approximate period of nonlinear oscillators with discontinuities by modified Lindstedt-Poincare method. Chaos, Solitons and Fractals, 23, 577-579. https://doi.org/10.1016/j.chaos.2004.05.004

Nayfeh, A. H. (1973). Perturbation Methods. New York: John Wiley \& Sons.

Nayfeh, A. H., \& Mook, D. T. (1995). Nonlinear oscillations. New York: John Wiley \& Sons.

Razzak, M. A. (2016). An analytical approximate technique for solving cubic-quintic Duffing oscillator. Alexandria Engineering Journal, 55, 354-364. http://dx.doi.org/10.1016/j.aej.2016.04.036

Sanchez, N. E. (2005). A view to the new perturbation technique valid for large parameters. Journal of Sound and Vibration, 282, 1309-1316. http://dx.doi.org/10.1016/j.jsv.2004.09.030

Shariyat, M., \& Dabari, E. (2013). A variational iteration solution for elastic-plastic impact of polymer/clay nanocomposite plates with or without global lateral deflection, employing an enhanced contact law. International Journal of Mechanical Sciences, 67, 14-27. http://dx.doi.org/10.1016/j.ijmecsci.2012.12.003

Zhang, H. L. (2009). Periodic solutions for some strongly nonlinear oscillations by He's energy balance method. Computers and Mathematics with Applications, 58, 2480-2485. http://dx.doi.org/10.1016/j.camwa.2009.03.068

Zhang, X., Zou, L., Liang, S., \& Lui, C. (2018). A novel analytic approximate method with a convergence acceleration parameter for solving nonlinear problems. Communications in Nonlinear Science and Numerical Simulation, 56, 1581-1585. http://dx.doi.org/10.1016/j.cnsns.2017.08.025

\section{Copyrights}

Copyright for this article is retained by the author(s), with first publication rights granted to the journal.

This is an open-access article distributed under the terms and conditions of the Creative Commons Attribution license (http://creativecommons.org/licenses/by/4.0/). 